\title{
A Novel Multipath Routing Ring Protocol Adapted for WMSN
}

\author{
B.M. Taj, M. Ait Kbir \\ Faculty of Sciences and Technology, Laboratory LIST, University of Abdelmalek Essadi, \\ l'Aéroport, Km 10, Ziaten. BP: 416, Tangier, 60000, Morocco
}

\begin{tabular}{l}
\hline \hline Article Info \\
\hline Article history: \\
Received Jun 6, 2018 \\
Revised Jul 21, 2018 \\
Accepted Sep 21, 2018 \\
\hline
\end{tabular}

Keywords:

Castalia

GPSR

Multipath routing ring

MWSN

Pyramidal technique

\begin{abstract}
In this paper an enhanced protocol of multipath routing ring that is suitable for transferring images by making many upgrades. First, instead of using propagation between the layers, we use certain parameters such distance and delay to pick the next node of the lower layer. Then, we used an heterogeneous network, including sensors (capture and send images) and connectors (serve as a bridge to the sink). Finally, we used the pyramid decomposition technique. To avoid congestion, we adjust the number of levels based on the state of the network. To verify our protocol, we used Castalia simulator to simulate the real transmission conditions. Then, we compared it with GPSR protocol and the original Multipath routing ring protocol. Our proposed protocol proves its efficiency by transferring more images with better quality (PSNR) and consuming less energy compared to other protocols.
\end{abstract}

Copyright $\odot 2018$ Institute of Advanced Engineering and Science. All rights reserved.

\section{Corresponding Author:}

B.M. Taj,

Faculty of Sciences and Technology,

Laboratory LIST, University of Abdelmalek Essadi,

l'Aéroport, Km 10, Ziaten. BP: 416, Tangier, 60000, Morocco.

Email: bennani.taj@gmail.com

\section{INTRODUCTION}

The rapid development in the micro-electro mechanical system (MEMS) has made possible the creation of miniaturized and affordable sensors. These miniaturized sensors communicate wirelessly. A sensor is a combination of multiple components like transmission unit, power unit control, processing unit and control unit [1]. In addition to the basic components, a sensor can be equipped with special sensors which can sense and measure the surrounding environment such as temperature, distance, pressure etc., and transform them into digital values. The acquired data are stored or transmitted based on the need. Nevertheless, the limitation of a sensor is a big issue for many reasons. First, there is a limitation in the processing power which affects processing of data. Then, the limited energy affects the amount of stored data. Finally, the limitation in term of energy affects the lifetime duration of sensors [2].

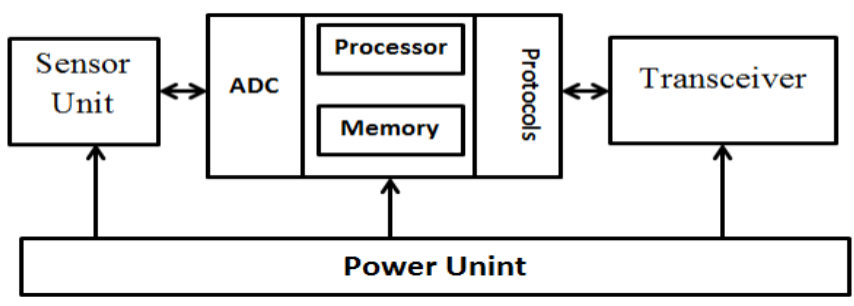

Figure 1. Structure of a sensor 
A network of linked sensors (hundreds or thousand nodes in a large area) forms a wireless sensor network (WSN). In fact, it covers a large area and used in multiple areas: military, medical, industrial monitoring or environmental phenomena, etc. The acquired data are transmitted from one sensor node to another until they reach the sink (base station) [1]. This sink is an exit to a remote external device (e.g. the internet). This device can be used for storing, analyzing and processing data as shown in the Figure 2 [2]/

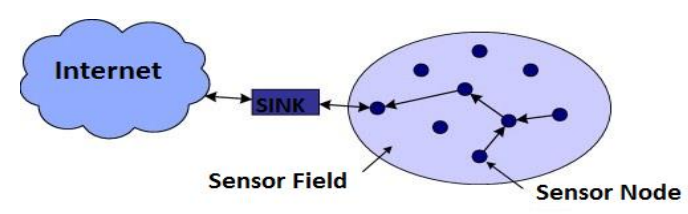

Figure 2. WSN architecture

On the contrary of WSN which can transmit only scalar data, WMSN can transmit more sized and complex data like multimedia content. In MWSW, sensor nodes are equipped with sensors that can capture multimedia content. This kind of network has several structures [3] including the single-tier flat, the singletier clustered and multi-tier. In our paper, we applied the second structure by using sensors (capture and transmit in ages) and connector (simple nodes serving as a bridge). The Figure 3 depicts the architecture that we used. In fact, WMSN requires some QOS demands. These demands could be the highest bandwidth demand, the use of cross-layer, the limit of resources (energy, memory, etc...) and the coding of multimedia content [3].

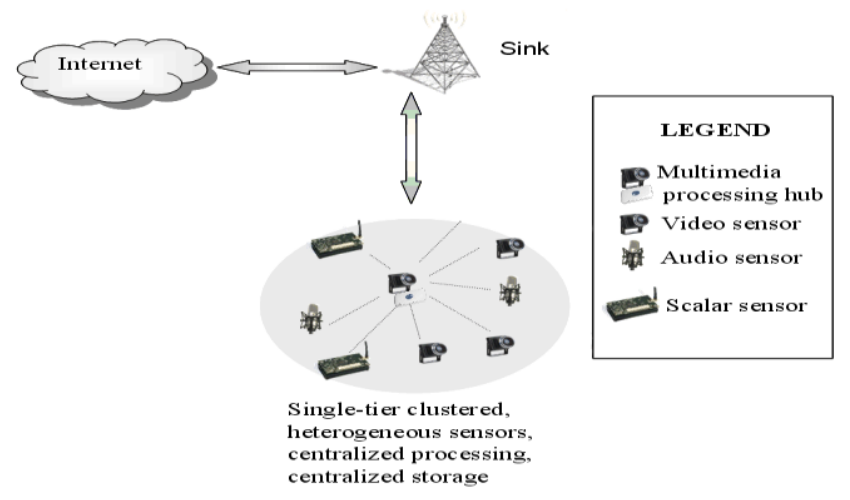

Figure 3. Used wireless multimedia sensor network architecture

When it comes to process images, many methods exist. The pyramid method [4] is one of the most efficient techniques. It uses the Gaussian. The Gaussian pyramid is calculated as follows. The original image is processed with a Gaussian kernel. As a result, we get an image with a low pass filtered version of the original image. The Figure 4 shows this process.

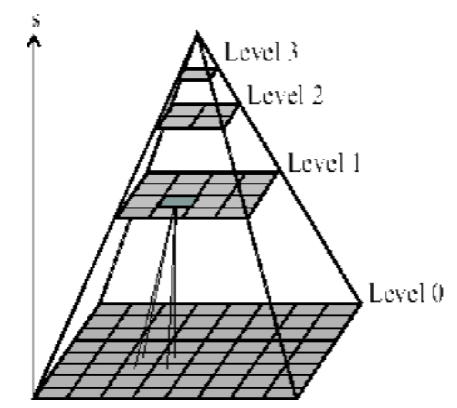

Figure 4. Gaussian and Laplacian pyramids 
In WMSN, many layers interfere in the transfer process like radio layer, Mac layer, application layer and especially the routing layer. Routing protocols plays a major role in managing the limited resources and delivering images in good quality at the same time. Many routing protocols exist like clustered routing protocol and multipath routing protocol. In clustered protocols, nodes are grouped into a set called clusters. Each cluster has a cluster-head with which all other members communicate. It is the only node in the cluster that is allowed to send data out of the cluster. Many protocols in the same category exist, such as Teen [5], M-Leach [6], LEACH [7] etc. Multipath protocols consist of using different nodes between the source and the sink. The road to take can be static or dynamic depending on the routing protocol. There are multiple protocols like DD [8], SPIN [9], SAR [10], GPSR [11], multipath routing ring [12], etc. In this work, We are interested in multipath routing rowing. This special protocol, build layers starting from the sink, and each node belongs to a layer. The intermediate nodes are chosen between the lower layers until arriving to the sink.

During This paper, we are going to have different sections. First, we are going to present the related works. Then, we will detail our protocol. After that, we are going to explain and analyze the results of simulations that we made. Finally, we will finish our paper with a final conclusion.

\section{RESEARCH METHOD}

The most important thing in WSN is transferring data to the sink. Actually, one of the big challenges that we face is the distance between nodes because of the limited transmission range of nodes. Multipath routing protocols can solve this issue. Multiple protocols already exist like GPSR and Multipath routing ring.

\subsection{GPSR Routing Protocol}

The Greedy Perimeter Stateless Routing algorithm Consists of forwarding packets using multiple methods. At first, we use the greedy forwarding to deliver packets to the closest node. To transmit the packet to the next node, we use the position (origin and the destination of the packet) and the local optimal greedy.

In the first phase Of GPSR, we use the origin and the destination of the packet for forwarding it to the next hop using the local optimal greedy. Any sensor node knows the radio position of his neighbors. The next hope is picked based on destination's position. This process is repeated until the packet gets to its destination.

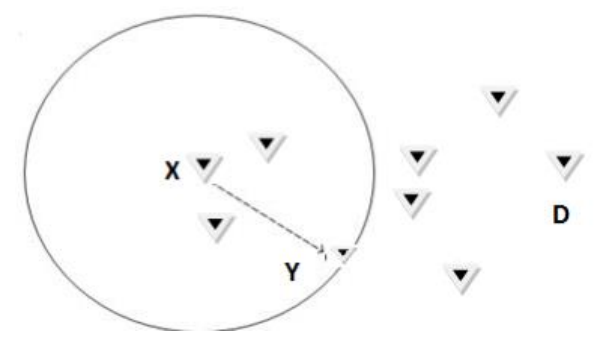

Figure 5. Example of greedy forwarding.y is the closest point of $\mathrm{x}$ neighbers forward

The Figure 5 shows an example of the greedy next hope choice. In the figure, the sensor node $\mathrm{X}$ sends a packet to $\mathrm{D}$, and the range of $\mathrm{X}$ appears in a circle. The arc with radius is equal to the distance between $\mathrm{Y}$ and $\mathrm{D}$. The sensor node $\mathrm{X}$ transmits the packet to $\mathrm{Y}$ if the distance between $\mathrm{Y}$ and $\mathrm{D}$ is less than the distance between $\mathrm{D}$ and any other sensor node. This process is repeated until the packet reached D.

The previous algorithm is used by sensor nodes to get information of all its neighbors. This update process is repeated periodically. During this process, all nodes transmit their information (ID, position, etc.) to the network.

If a node is not receiving a beacon as a response from a neighbor for longer than timeout interval, a GPSR router will consider the neighbor as dead or gone out of range, and removes the neighbor from its table. The 802.11 mac layer indicates the link level of retransmission failures to neighbors.

The advantage of grade forwarding is the dependence only on the knowledge of the forwarding node's immediate neighbors. In this step, the density of nodes is more important than other things like the number of nodes. In this process, we use multi-hop. Also, the total number of nodes must be higher than the number of the neighbor's node. Once this process fails, we use the perimeter forwarding method. 


\subsection{Multi-Path Routing Ring}

Multipath routing ring is a routing protocol that is using the notion of layers around the sink, and each layer container node). The distance from the sink determines the level of the layer starting from 0 (sink). This protocol follows two steps: construction phase and the sending phase.

In the first step The sink node creates the layers by broadcasting the topology setup packet. The nodes which receive it belong to layer 1 . Then, the nodes that belong to level 1 Broadcasts the same packet. Nodes receiving the packet form layers. This process is repeated until all nodes have a layer level. After this step ends, the transmission step begins. The Figure 6 depicts the formation of layers.

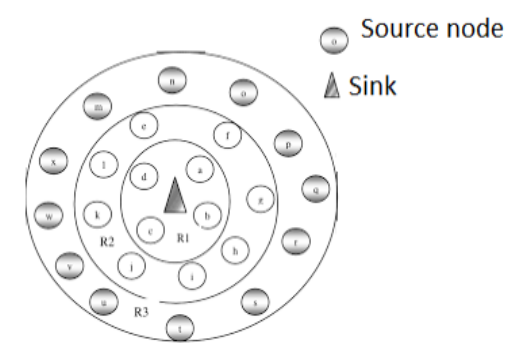

Figure 6. Nodes organized in layers

Many ways of transmitting packets from higher layers exist.In [13] they propose an algorithm in which a routing table is not needed. When a node wants to transmit a packet, it broadcasts it to the lower level. Then, the intermediate node does the same thing. This process is repeated until the packet gets to the base station.

\section{PROPOSED PROTOCOL}

In this paper, we are going to propose an improved multipath routing ring routing protocol, which is adopted to image transfer. Our proposed protocol Consumes less energy and can transfer more images with a better quality. Actually, original multipath routing ring protocol uses broadcast between layers to transmit packets and doesn't use any routing table, which has multiple drawbacks. First, the energy consumption is high because of the repeating broadcasting process. Second, the propagation of packets provokes a collision of packets. Finally, this protocol suffers from a severe loss of packets which affects the number of received images. In order to solve these problems, we proposed a heterogeneous architecture based on sensors and connectors. Also, the transmission process is based on routing table which minimizes the traffic in each layer. Finally, the image compression ratio is adaptable in function of the state of the network.

\subsection{Construction Phase}

At first, the layer ID of all nodes is -1. Also, A node contains two routing tables (one for lower connectors and one for lower sensors) which are initially empty. The formation of layers starts from the sink by broadcasting a construction packet. This packet contains the position of the sink. The both kinds of nodes that receive the packet form the layer 1 . Also, they store the position of the sink and the delay from the sink. Then, the nodes of layer 1 broadcast the construction packet again. The nodes that receive this packet form the layer 2. The layers that are upper than 1 store data about all possible paths from the sink including the total distance from the sink, the total delay from the sink, the RSSI of the lower node, the distance from the lower node and the remaining energy of all the nodes of the path. If a node receives a packet from a sensor , it stores the information about the path in the sensor routing table otherwise it store them in connector routing table. Then, the nodes of layer 2 broadcast the construction packet to form upper layers. The same proeess is repeated again and again until all nodes belong to their corresponding layer. The Figure 7 shows the network after formation. Then two phases begin: update phase and transmission phase. 


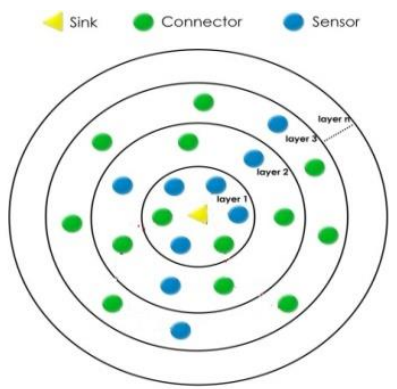

Figure 7. Connectors and sensors formed in layers

\subsection{Transmission Phase}

During this phase, sensor nodes transmit their data toward the sink. In our protocol, sensor nodes capture images and send them to connector nodes. In other hand, connectors serve as a bridge throughout the different layers until the sink. All nodes of layer 1 send their packets directly to the sink by adjusting the transmission power in fonction of the sink's RSSI. The sensor nodes of a layer $n$ transmit their data to the connectors of the lower (n-1). Before sending any packet, the selection of the next node follows the Figure 8 . Thus, the next rode can change depending on the traffic and packets can follow different paths as shown in Figure 9.

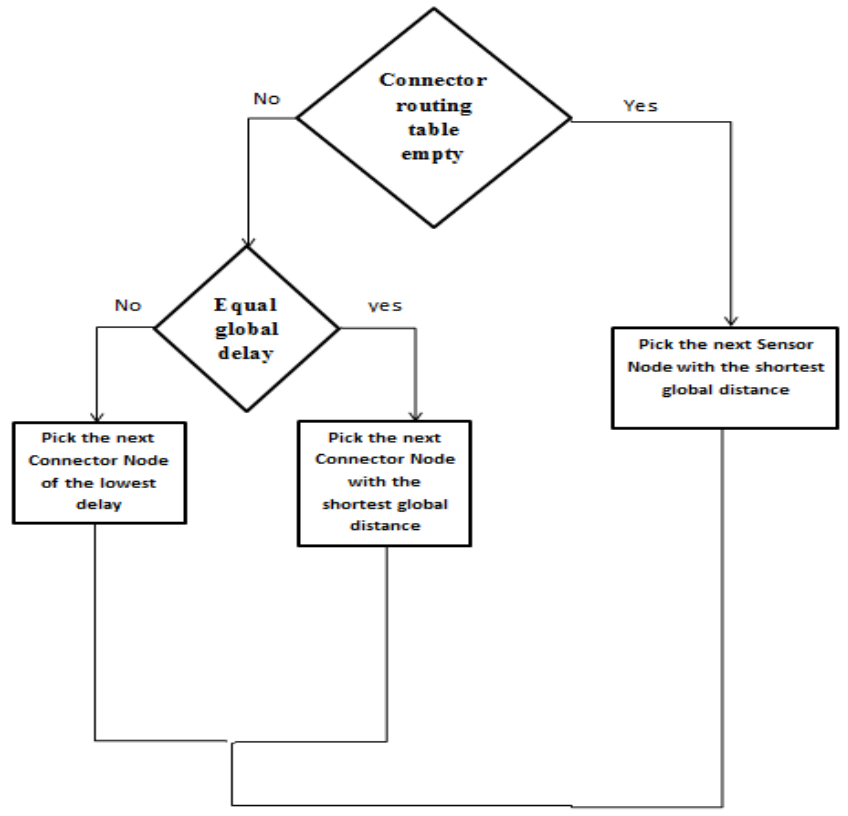

(a)

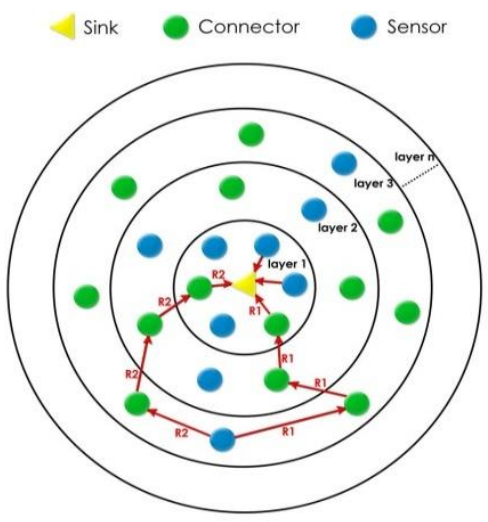

(b)

Figure 8 . The process used to select the next node

\subsection{Update and Image Compression}

This step starts after the formation phase, and it is an essential part of our protocol. It helps to find constantly the path which has the least traffic. The update process uses periodic and permanent way. Each period of time, the sink starts this process, and it goes from layer to layer. Second, when a connector node transmits a packet, it updates its relative information in the nodes of the upper layer. Actually, we update essentially the delay and the remaining energy. Another feature of our protocol is the variant compression ration based on the delay. Each period of time, the routing protocol calculates the number of levels based on the throughput of the link between the sensor and the sink. As shown in Table 1, more the delay is short less the compression is. Then, the calculated value is transmitted to application layer using The cross layering technique. As a direct effect, we control the size of the packet following the state of the network. 
Table 1. Number of Level in Pyramidal Technique in Fonction of Debit

\begin{tabular}{cc}
\hline Debit of the link in Kbps & $\begin{array}{c}\text { Number of } \\
\text { levels }\end{array}$ \\
\hline$>=100$ & 1 \\
$100>\ldots>=75$ & 2 \\
$75>\ldots>=50$ & 3 \\
$50>\ldots>=25$ & 4 \\
\hline
\end{tabular}

\section{RESULTS AND DISCUSSION}

\subsection{Proposed Applications}

In order to evaluate our protocol, we created a multimedia application in Castalis simulator. This application uses Opencv to compress and decompress images by applying multiple levels in pyramidal compression technique. Also, varying the time of sending images by using a timer. In our application, we follow multiple steps. First, the sensor nodes capture the gray image image. Then, the captured image is treated by using the Gaussian and Laplacian transforms. After that, we divide the obtained image on blocks of $8 \times 8$. Finally, we apply RLE/Huffman process as shown is Figure 10 .

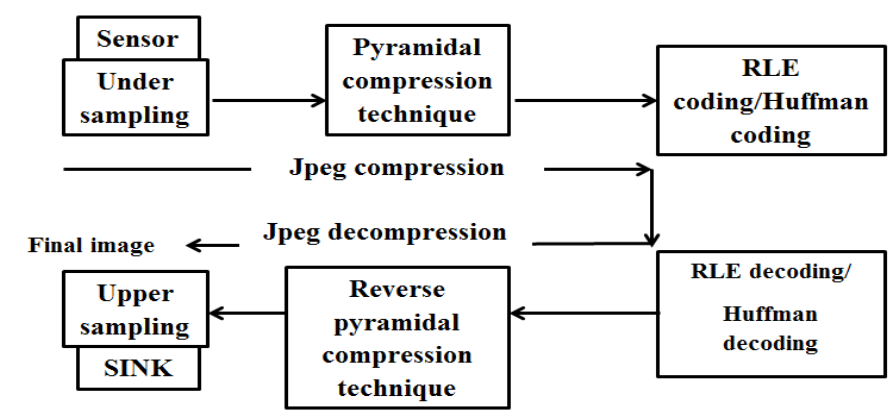

Figure 10. Compression/decompression process of JPEG image using the pyramidal compression technique

\subsection{Simulation Parameters}

In our paper, we used a distribution of 200 nodes over $200 \times 200 \mathrm{~m} 2$ as shown in the Figure 11. The Table 2 shows the parameters used in our simulations. The Figure 12 shows the original image.

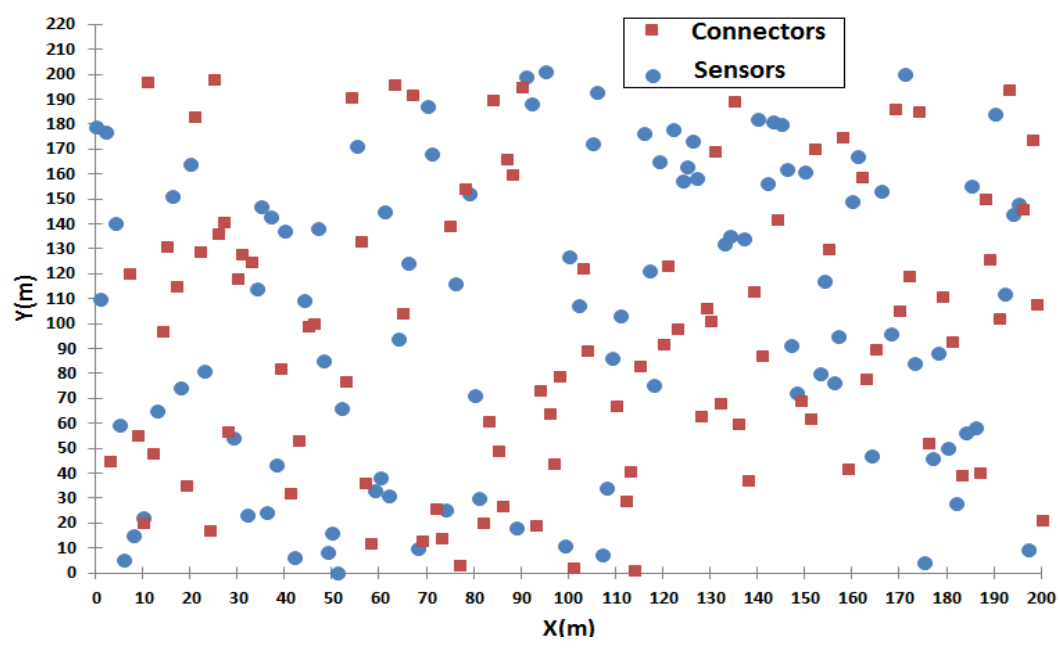

Figure 11. Distribution of nodes in the space 
Table 2. Simulation's parameters

\begin{tabular}{lc}
\hline \multicolumn{1}{c}{ Parameter } & Value \\
\hline Tpology size & $200 \times 200 \mathrm{~m} 2$ \\
Simulation time & $2600 \mathrm{~s}$ \\
Number of nodes & 200 \\
Period between sending packets & $0.1 \mathrm{~s}$ \\
Image compression & $90 \%$ \\
Number of trials & 20 \\
Initial power & $400 \mathrm{j}$ \\
BS position & $(0,0)$ \\
Percentage of sensors & $50 \%$ \\
Percentage of connectors & $50 \%$ \\
MAC protocol & Tunable MAC \\
\hline
\end{tabular}

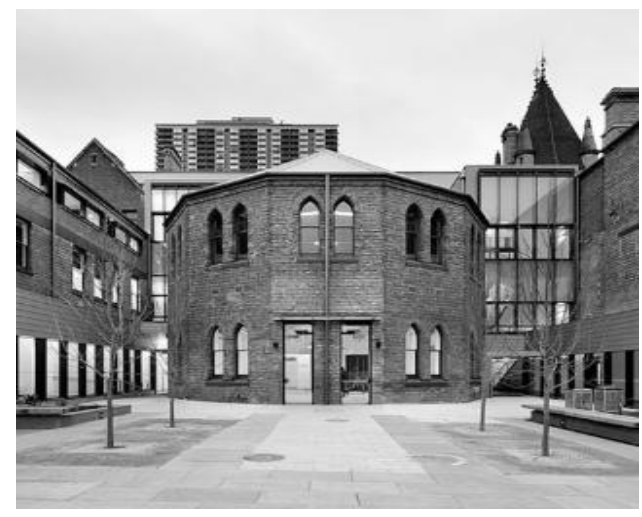

Figure 12. Original image to be sent

\subsection{Discussion Results}

To evaluate our proposed routing protocol, we compared it with GPSR and multipath routing ring routing protocol. Also, we used three approaches. First, we assess the energy efficiency by calculating. The energy consumption of the hall network per time. Second, we evaluate the multimedia efficiency by getting the completed images by the sink in function of time. Finally, we used the PSNR of receiving images to measure their quality. Actually, we measure the number of images in function of their corresponding PSNR.

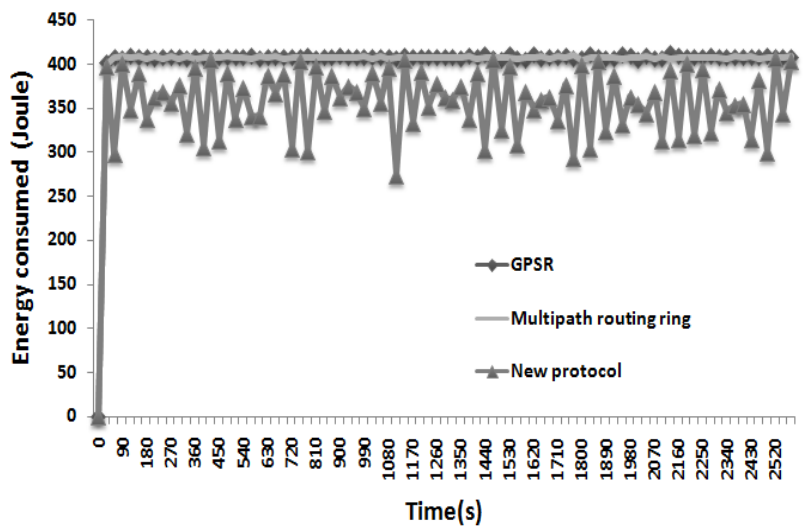

Figure 13. Energy consumption per time

The Figure 13 represents the energy consumption per time for all nodes. It shows a comparison between PSNR, multipath routing ring and our protocol. The graph shows that both GPSR and Multipath routing ring consumes nearly the same high level of energy. Also, it shows that our protocol consumes by far less energy than the other protocols. These results could be explained by many reasons. First, the GPSR 
routing protocol uses greedy perimeter and the perimeter forwarding methods. These methods make a lot of transmissions, which means high energy consumption. Also, the constant update for neighbors make the nodes consumes more energy. Second, the multipath routing ring uses the broadcast to transmit data from layer to layer until the sink. Thus, all nodes of the network consume a lot of energy. When it comes to our protocol, it consumes less energy for many reasons. First of all, every transmission energy is adjusted to the distance of the targeted node. Then, we don't use the broadcast as a method, but a packet must be received by a specific connector. Also, the use of heterogeneous nodes by using a percentage of nodes to transmit the data called connectors. The rest of nods Are called sensors, and they turn to sleep state when they deliver the image. After a while, they become active to deliver another image, and the cycle is repeated again and again.

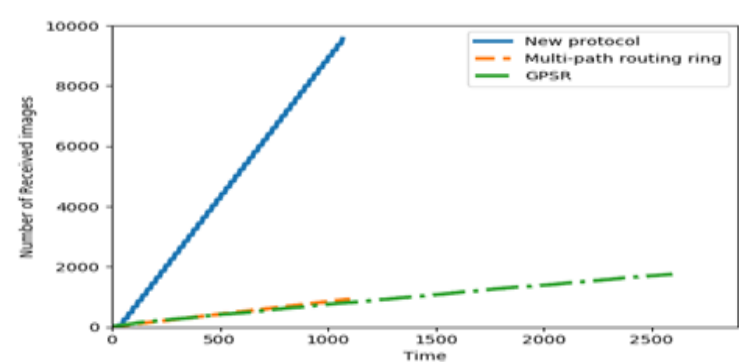

Figure 14. Number of received image as function of time

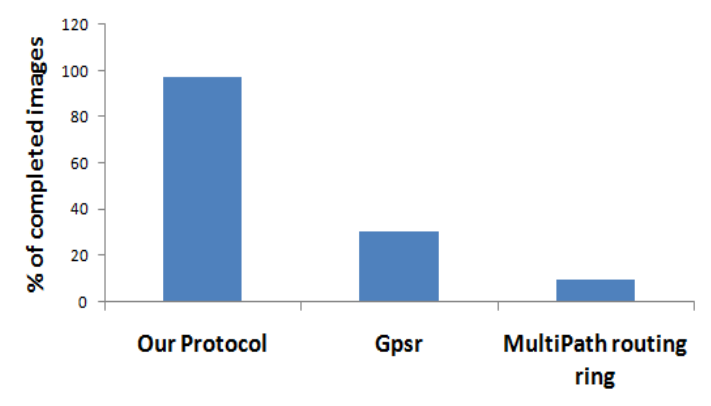

Figure 15. percentage of received completed image as function of protocol

The Figure 14 is a graph that represents the number of completed images by the sink per time. It reflects the multimedia efficiency of the three protocols. In this figure, we can see clearly That our protocol is more efficient than other protocols. These results are confirmed by the graph of Figure 15 which shows the percentage of completed images. It shows that our protocol is the most efficient and multipath routing ring is the least efficient. In fact, we can explain these results with many reasons. First, GPSR uses intermediate nodes by applying a Greedy Perimeter method constantly. Since the density of nodes affects GPSR routing protocol, the number of intermediate nodes increases. The delivered packets to the sink decrease, which affects The image constructed by the sink. On the other side, Multipath routing ring suffers from a severe problem which is the broadcast. Actually, when a sensor node sends a packet, it can be received in full range of transmission causing the collision of packets. This problem reduces considerably the amount of the received packets by the sink and the number of completed images is less. Finally, our protocol has multiple features that make it more efficient. First, it uses layers, which limit the number of intermediate nodes. Thus, the packets arrive quickly to The sink. Second, the choice of the next node is based on delay and distance. So, it can avoid congestion. Third, using the delay as a criteria to choose the compression ratio makes us control the size of the packets. Actually, the average of the compression ratio is $10 \%$, which proves the efficiency of our protocol. Finally, Using connector nodes to conduct the packet to the sink, diminishes the collision zones and congestion. These features make our protocol more suitable for WMSN transfer.

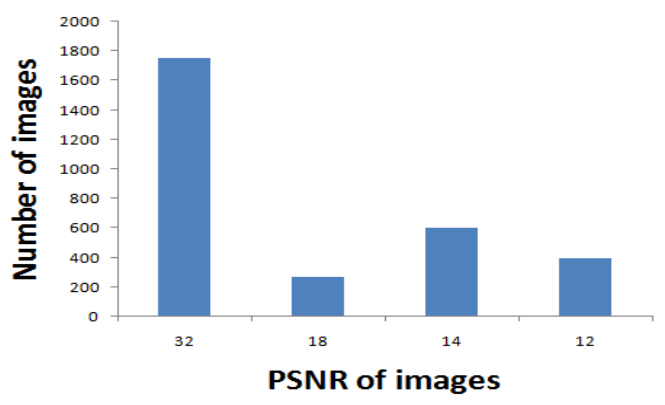

Figure 16. Number of received images per PSNR using GPSR

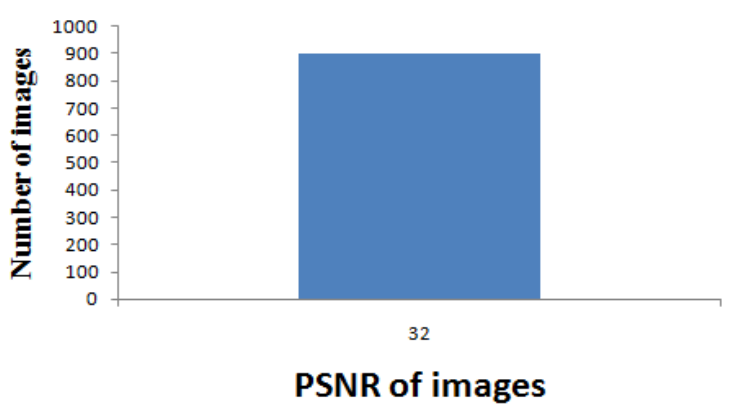

Figure 17. Number of received images per PSNR using Multipath routing ring 


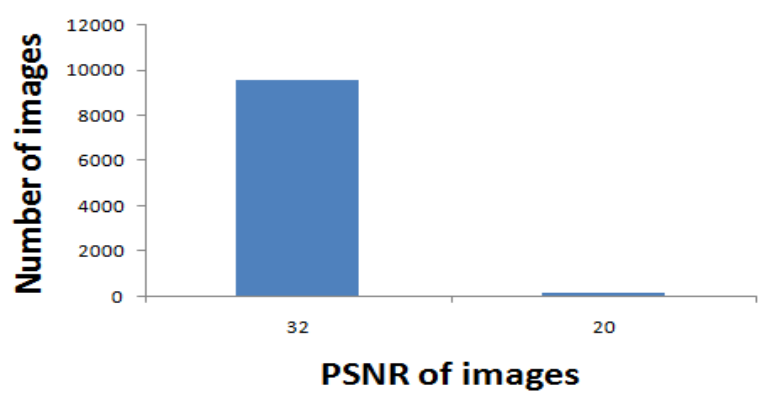

Figure 18. Number of received images per PSNR using our protocol

The Figures 16, 17 and 18 show the total number of imager per PSNR quality. According to the Figure 17, Multipath routing ring has the minimum received images even if they are completed. Thus, the number of lost images is high, which is 9100 images. In the Figure 16, GPSR delivers a large variety images from lower quality to complete images. Although, the number of lost images is 6976 images, which is important. Finally, the Figure 18 shows that our protocol is near to $100 \%$ of efficiency by $99 \%$ of complete images and 0 lost images. These results confirm the previous results making our protocol the best among the three protocols.

\section{CONCLUSION}

In this paper, we made an application that compress/ decompress images using the Gaussian and the Laplacian transforms, And the number of levels depends on the state of the network. Then, the compressed images are delivered to the network and decomposed at the sink. Actually, we used this application to test the efficiency of our protocol in term of multimedia content transmission. We can deduce that our protocol is more efficient by delivering images in good quality and big quantity. Also, we proved that it consumes less energy. We conclude that our proposed protocol is more suitable for the MWSN compared to other protocols. Although, it could be optimized to transfer more complex multimedia content like videos.

\section{REFERENCES}

[1] Estrin D, Culler D, Pister K, and Sukhatme G. Connecting the physical world with pervasive networks. IEEE Pervasive Computing. no. 1. January 2002; Vol. (1) 59-69.

[2] Rahman K C. A survey on sensor network. Journal of Computer and Information Technology. no. 1, 2010; Vol. (1) 76-87.

[3] Abazeed M, Faisal N, Zubair S, and Ali A. Routing Protocols for Wireless Multimedia Sensor Network: A Survey. Journal of Sensors. 2013; 1-11.

[4] Derpanis K G, The Gaussian Pyramid. 2005.

[5] Manjeshwar A and Agrawal D P.TEEN: ARouting Protocol for Enhanced Efficiency in Wireless Sensor Networks. Proc. of the 15th International Parallel \& Distributed Processing Symposium. Washington DC. USA. 2001; 189.

[6] Kodali R K, S. Bhandari V S K A, and Boppana L. Energy efficient m- level LEACH protocol. Proc. International Conference on Advances in Computing. Communications and Informatics (ICACCI). 2015; 973-979.

[7] Heinzelman W R, Chandrakasan A, and Balakrishnan H. Energy-Efficient Communication Protocol for Wireless Microsensor Networks. Proc. of the 33rd Hawaii International Conference on System Sciences. Washington DC. USA. 2000; 8:8020 .

[8] Intanagonwiwat C, Govindan R, Estrin D, Heidemann J, and Silva F.Directed diffusion for wireless sensor networking. IEEE/ACM Transactions on Networking. February 2003;11: 2-16.

[9] Heinzelman W R, Kulik J, and Balakrishnan H.Adaptive Protocols for Information Dissemination in Wireless Sensor Networks. Proc. of the 5th Annual ACM/IEEE International Conference on Mobile Computing and Networking. New York. USA. 1999; 174-185.

[10] Sohrabi K, Gao J, Ailawadhi V, and Pottie G J.Protocols for self-organization of a wireless sensor network.IEEE Personal Communications. no 5. October 2000; Vol. (7) 16-27.

[11] Karp B and Kung H T.GPSR: Greedy Perimeter Stateless Routing for Wireless Networks.Proc. of the 6th Annual International Conference on Mobile Computing and Networking. New York. NY. USA. 2000; 243-254.

[12] Huang G M, Tao W J, Liu P S, and Liu S Y, .Multipath ring routing in wireless sensor networks. in Applied Mechanics and Materials. 2013; Vol. (347) 701-705. 